\title{
Déjà vu and other dissociative states in memory
}

\author{
Akira R. O'Connor, Christine Wells \& Chris J. A. Moulin
}

To cite this article: Akira R. O'Connor, Christine Wells \& Chris J. A. Moulin (2021)

Déjà vu and other dissociative states in memory, Memory, 29:7, 835-842, DOI: 10.1080/09658211.2021.1911197

To link to this article: https://doi.org/10.1080/09658211.2021.1911197

册 Published online: 09 Aug 2021.

Submit your article to this journal 주

Џlll Article views: 533

Q View related articles $\longleftarrow$

View Crossmark data $\asymp$ 


\section{Déjà vu and other dissociative states in memory}

\begin{abstract}
Though we know little more about déjà vu today than we did back in the days of Arnaud over a century ago, we have learned a great deal about other forms of misattribution. This is hard-won knowledge with potentially vital consequences for society: misattribution can alter our lives in strange and unexpected ways. Schacter (2002, p. 91)
\end{abstract}

In recent years, déjà vu has become of great interest in cognition, where it is mostly seen as a memory illusion. It can be described as having two critical components: an intense feeling of familiarity, and a certainty that the current moment is novel. As such, déjà vu can be described as a dissociative experience, resulting from a metacognitive evaluation (the certainty) of a lower-level memory process (the familiarity). In emphasising this dissociation we are deliberately aligning the experience with other instances where metacognitive or subjective processes become divorced from the current goals of processing. For example, jamais vu ("never seen") is often operationsationalised as being the reverse of déjà vu; the subjective experience of unreality or unfamiliarity for a stimulus known to be familiar.

Déjà vu has variously been described as a misattribution (captured in the quote above; Schacter, 2002); an illusion (Jacoby \& Whitehouse, 1989; Penfield \& Perot, 1963); and a cognitive or "epistemic" feeling (da Sousa, 2009; Moulin \& Souchay, 2014). Here we describe such phenomena as dissociative since they arguably all converge on the idea that a "feeling" or "experience" becomes dissociated from a process, as has been argued for other subjective experiences, such as the Tip-of-the-Tongue state (Schwartz \& Metcalfe, 2011). This dissociative account of such subjective experiences is inherently metacognitive: these phenomena are dissociative in that they signal the existence of a metacognitive evaluation or feeling which has become detached from the ongoing mental operation. In the case of the tip-of-the tongue, for instance, there is a feeling of knowing experienced for currently unretrievable information.

The entirety of experiences related to déjà vu is less easy to define, because other terms are less often used, and there is less consensus in their definition. These experiences include jamais vu (defined above), presque $\mathrm{vu}$, the feeling of an imminent profound insight or epiphany; and prescience, the feeling of being able to predict the future, which has been proposed in the literature to be both separate from déjà vu or a part of it. In order to conform with our proposal about conflicts between what is known and what is felt, we suggest that prescience and presque vu should refer to dissociative states. In prescience, the feeling of sensing of the future soon subsides, and the experient realises that it is not possible to have known the future, i.e. the feeling is eventually (or perhaps immediately) known to be false. Likewise, in presque vu, it is not that we have actually had a profound insight, but we feel that we have had a profound insight, and we know that we have not. Thus a defining characteristic of déjà vu and related states is that they refer to conflictual, erroneous interpretations of processing. déjà vu is not simply finding something familiar. The "eureka" moment in problem solving, if felt when actually solving a problem, is not presque vu, as examples.

Our hypothesis is that these experiences are all critical for understanding how subjective states guide our cognitive processing. In the case of déjà vu, the idea is that it exposes metacognitive mechanisms at play in the interpretation of familiarity signals, and as such theories of episodic memory should be able to accommodate this infrequent experience. Jamais vu possibly helps us understand the relationship between meaning and familiarity (and can be hypothesised as being related to the concept of semantic satiation). Presque vu possibly, by analogy, indicates the role of subjective experiences in decision-making. Finally, given the idea that episodic memory is a concept which is seen as aiding in generating representations of the individual in both the future and the past, it seems a logical possibility, that false feelings of familiarity and remembering can indeed produce projections into the future. Because of the presence of déjà vu and associated phenomena in several pathologies, it seems a clinical imperative to better understand this fleeting experience.

\section{Definition of Déjà vu}

The term déjà vu comes from the French, literally, "already seen". It is distinguished from false memory by the fact that the experiment is aware of a conflict in mental evaluations; in the déjà vu experience, the experient is aware that the feeling of familiarity is not logical, possible, or is false. In everyday language, two possible usages of the phrase exist, one which is consistent with the scientific 
usage, and one which refers to monotonous, unusual or annoying repetition. Historically in the scientific literature, déjà vu was firstly used to refer to any feeling of having encountered information before whether it was correct or not, thus to speak of recognition memory in amnesia, early theorists would discuss whether the sense of déjà vu was preserved or not (e.g. Claparede). Particularly through its use in parapsychological texts, the term gradually came to mean a false feeling of familiarity. Early authors (e.g. Arnaud, 1896; translated by Bertrand et al., 2017) noted that the use of the term was plagued by ambiguities and inconsistencies, and it was not until Neppe (1983, p. 3) that the field converged on a common definition of the term, albeit in a parapsychological work: "Any subjectively inappropriate impression of familiarity of a present experience with an undefined past."

The term has most consistently and rigorously been applied to experiences in epileptic populations where it was described as early as C19, summed up by William James as the "sudden invasions of vaguely reminiscent consciousness", but without explicitly using the term déjà vu. Citing this early work, and attempting to refine further the concept of "dreamy states" in epilepsy, Penfield (e.g. Penfield \& Perot, 1963) used the term déjà vu uniquely to describe the illusory sensations of familiarity observed both spontaneously and under electrical stimulation in temporal lobe epilepsy. Subsequent works have attempted to differentiate déjà vu in healthy and epileptic populations using questionnaires and subjective reports, concluding that the healthy and pathological forms of the experience are indistinguishable (e.g. Warren-Gash \& Zeman, 2014).

\section{Progress in Déjà vu Research}

Shortly after Schacter published The Seven Sins of Memory, from which the quote above is taken, Brown published two seminal reviews: a paper in Psychological Bulletin (Brown, 2003) and a book (Brown, 2004) in which he presented a comprehensive review of the literature in a range of domains. A common thread through the literature was the reliance on non-experimental questionnaire research. As such, déjà vu was presented as a phenomenon with demographic and situational correlates, but with little known of its cause or mechanisms. Brown's reviews were a rallying cry to experimental and neuropsychological researchers, catalysing new interest in the study of the déjà vu experience.

A key to this new interest was the originality of experimental approaches and methodological techniques used by researchers. Brown himself was involved in experimental work generating illusions of past encounters (Brown \& Marsh, 2009), whilst the Cleary and O'Connor groups extended their work on subjective experiences of memory to include experimental analogues of déjà vu (e.g. Cleary, 2008; Urquhart \& O'Connor, 2014). Neuropsychological work progressed to classify varieties of déjà vu-like experience associated with pathological ageing (Moulin, 2013), epilepsy (Illman et al., 2012; Martin et al., 2012). and a range of more unusual antecedents (including pharmacologically induced déjà vu, e.g. Kalra et al., 2007; Singh, 2007). The growing availability of neuroimaging facilities allowed researchers to gain insight into potential structural brain correlates of the experience (e.g. Brázdil et al., 2012). Developments in the neuroanatomical basis of déjà vu also continued to draw upon the stimulation techniques first pioneered by Penfield, especially in the Chauvel group (e.g. Bartolomei et al., 2012).

The growth of the déjà vu research ecosystem into combinations of retrospective, neuropsychological, experimental and imaging approaches is represented well in this special issue and, most importantly, broadens the potential of the field to contribute new understanding of the experience. Such was the range of work published in the decade after Brown's reviews, that in the lead up to the 2016 International Conference on Memory (ICOM) in Budapest, the editors of this special issue judged there to be critical mass in the research community to sustain a symposium dedicated to déjà vu (see Figure 1). Indeed, at that symposium, Dan Schacter acted as the Discussant, closing the session with a positive reappraisal of the progress made in the realm of déjà vu research.

\section{This Special Issue}

All of the speakers at the ICOM symposium on déjà vu are represented in this Special Issue. We are also delighted to welcome contributions from authors who were in the audience but did not present at the symposium--a demonstration of the extent to which exposure to the study of déjà vu continues to propagate high quality research in the field. Here we outline each contribution to this special issue. These papers, unsurprisingly, also represent the research traditions in the literature, collecting together single-case and small group neuropsychological studies, large scale questionnaire studies, and experimental simulations or analogues of déjà vu (and other dissociative experiences). To present the articles in the Special Issue, we tackle each of these methodological approaches in turn.

Four articles in the current issue take a neuropsychological approach, and the first three here present single case studies. These studies are a continuation of the tradition of publishing case reports in déjà vu, stemming from Arnaud's description of Louis (Arnaud, 1896). Déjà vu is so infrequent and unpredictable an experience when it occurs naturally that it is somewhat natural that willing participants with unusual and more frequent presentation of déjà vu are targets for research studies. Curot, Pariente, Hupé, Lotterie, Mirabel and Barbeau (2021) present the case OHVR, and discuss the role of the hippocampus in déjà vu, attempting to introduce the concept of prescience into the classification of déjà vu vu states, drawing on the earlier work of Illman et al. (2012) who had proposed forms 


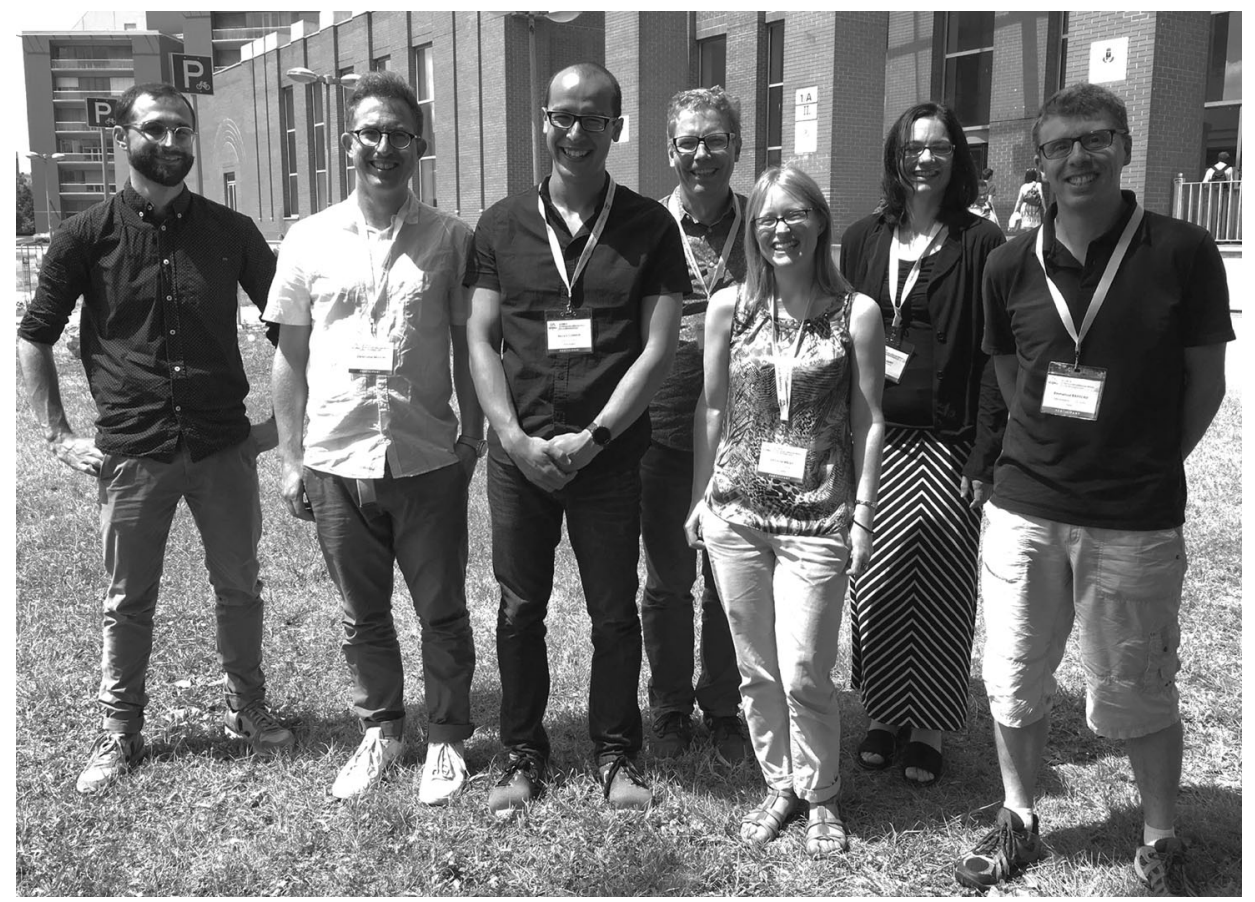

Figure 1. Speakers at the ICOM symposium on déjà vu, Budapest, 2016. From left to right: J. Curot, C.Moulin, A. O'Connor, S. Kohler, C. Wells, A., Cleary, E. Barbeau.

of déjà vu experience based on either familiarity or recollection (see Brandt et al.'s (2021) case below). OHVR articulately and spontaneously divided her dissociative experiences into déjà vu, a simple feeling of erroneous familiarity, and prescience, which she described as being able to predict the future. This latter experience was not unpleasant, and OHVR even stopped taking her medication in order to re-experience the sensation. A unique aspect of OHVR's case is that she is synaesthesic, something which warrants further investigation in reference to déjà vu more generally.

Curot et al. (2021) tackle a problematic issue in the déjà vu literature, that of the role of the hippocampus in the experience. The hippocampus has long been known to be a crucial structure in memory and novelty detection (e.g. Nyberg, 2005), but the literature to date has provided equivocal findings regarding whether the hippocampus is involved (and indeed what type of déjà vu it is involved in). Curot et al. (2021) do not solve this issue, but do point out that despite having markedly reduced hippocampal volume and intact parahippocampal structures, OHVR nonetheless experiences déjà vu several times a day. Cognitively, she has impaired recall but intact recognition, which may be conceived as the source of the déjà vu: she is able to find things familiar, but is unable to recollect why she finds things familiar, not unlike Cleary's concept of recognition without identification (Cleary, 2008).

Brandt and colleagues (Brandt, Conway, James and Oertzen, 2021) report a single case, patient MR, with mesio-temporal epilepsy associated with cavernoma in the left entorhinal cortex. The authors tested MR against controls using the Inventory for déjà vu Experiences Assessment (Sno et al., 1994) and experimental procedures, showing both elevated reports of déjà vu, and déjà vecu (which has been described as a form of déjà $\mathrm{vu}$ in which the experience is of recollection of a prior event rather than familiarity). Brandt and colleagues used these data to support the previously proposed contention that familiarity deficits caused by parahippocampal disruption lead to déjà vu, whereas more substantial memory deficits, in the form of recollective disruption, lead to déjà vecu (Illman et al., 2012).

Again, this work continues the historically fruitful investigation of déjà vu based on the experiences of people with epilepsy, offering insight into the distinctions between déjà vecu and déjà vu. That this distinction is investigated in the same patient is noteworthy, demonstrating a distinction between déjà vu and déjà vecu that is not based on clinical profile and based on subjective mnemonic experiences of familiarity and recollection. More generally, this work continues to stress the importance of single case studies both in the somewhat specialised study of déjà vu, but also in broader debates within psychology research, such as the theoretical single-/dual process recognition memory debate.

The third single case is presented by Ernst, Delrue and Willems (2021). They developed and tested an intervention aimed at reducing frequent déjà vu in a woman who suffered a cerebral haemorrhage in the right thalamo-callosal region, thereby improving her quality of life. The patient, 25 year-old MN, underwent a programme of psychoeducation--e.g. gaining insight into the causes of 
erroneous familiarity--and applied this knowledge to a better metacognitive evaluation of her subjective experiences of memory. The intervention was successful, reducing MN's reporting of déjà vu from once a day to once a week, and also led to improved performance in some standardised memory measures.

This work has potentially huge importance to those in the déjà vu research community who are frequently asked for advice on treating patients with persistent, frequent déjà experiences. The study outlines a promising intervention programme by which meaningful improvements in quality of life have been achieved in a patient, and paves the way for larger-scale testing in a range of samples in whom pathological déjà vu is more frequently observed. Additionally, the results reported here suggest that the cognitive monitoring within the déjà vu experience (which forms a central component of the Urquhart et al. (2021) paper in this issue) may be amenable to topdown control. From both applied and theoretical perspectives, this work once again illustrates the contribution of single cases to memory research.

Martin and colleagues' (Martin, Mirsattari, Pruessner, Burneo, Hayman-Abello and Köhler, 2021) also use a neuropsychological approach, but with a group of epilepsy patients. Their contribution is a continuation of their work examining the profile of recollection and familiarity responses for people with temporal lobe epilepsy who do and do not experience déjà vu (Martin et al., 2012). This previous research postulated that déjà vu during seizures in people with unilateral temporal-lobe epilepsy (UTLE) is related to their rhinal-cortex abnormalities, and manifests as recognition memory deficits that selectively affect familiarity. A number of researchers have interpreted this previous research as implicating recollection processes in the generation of "conflict" (e.g. Moulin et al., 2017), such that temporal lobe dysfunction leads to the generation of false familiarity, but intact recollection is required to reject this familiarity as false: “... TLE patients who experience déjà vu during their seizures, and who appear to have spared hippocampal functioning, exhibit a preserved ability both to make accurate recollectionbased recognition responses and to counteract familiarity signals with recovery of contextual information..." (Martin et al., 2015, p. 183).

The follow-up work presented in the current volume concerns bilateral temporal lobe epilepsy patients (bTLE), and fails to find the same pattern of performance across recollection and familiarity tasks; finding that the bTLE patients had impaired recollection and familiarity compared to controls. Whilst this detailed analysis continues to support the idea of the involvement of the rhinal cortex in déjà vu, the role of recollection processes is less clear. Neuroanatomically, the bTLE patients reported in the current issue had overall lower volumes of medial temporal lobe structures, and this difference was primarily due to reductions in the hippocampus. Summing across the article in this volume and the previous work, it appears interictal impairments of familiarity are relatively common in TLE, but that a combination of intact recollection and impaired familiarity is relatively rare. Moreover, this pattern of intact recollection and impaired familiarity is not the only pattern of performance in recognition tasks giving rise to high levels of déjà vu experience in TLE.

Where does this leave the idea that recollection is critical for generating the conflict inherent in the déjà vu experience? Martin et al. (2021) suggest that whereas recollection may be a source of information that leads to the "subjective inappropriateness" of the erroneous familiarity, it is possible that other information can be brought to bear to generate this conflict. That is, recollection is one source of information which may "oppose" or counteract the false familiarity in déjà vu. Other sources of information that could serve the same role include semantic knowledge, top-down evaluations of plausibility, and so on. That is, Martin et al. (2021) also suggest that the mnemonic conflict may be generated outwith the temporal lobe, citing Urquhart et al. (2021). They also point out that recent high-resolution neuroimaging studies have suggested that hippocampal subfields that process contextual novelty are possibly distinct from those responsible for recollection (for research on the possible role of hippocampal subfields in déjà vu see Pešlová et al., 2018). The study in the current issue was not able to analyse specifically such a fine-scaled account of the "mismatch" signals generated by the hippocampus, which clearly remains a priority for future research.

Only one study in this special issue represents the individual differences approach to studying déjà vu, where previous large scale questionnaire studies have examined déjà vu in healthy participants (e.g. Adachi et al., 2003), but also people with epilepsy or schizophrenia (e.g. Adachi et al., 2006). Wells and colleagues (Wells, O'Connor \& Moulin, 2021) take the same approach but for the first time consider people with anxiety. Their contribution is motivated by the need to better understand the contribution of anxiety to feelings of déjà vu. In part, the study was designed in order to test hypotheses generated in a case study presented in Wells et al. (2014). They report a 23-year-old man with a history of chronic anxiety and low mood who had an abnormal score on the Dissociative Experience Scale (DES; Bernstein \& Putnam, 1986) and who reported compulsive behaviours at times of stress, and also reported near constant feelings of déjà vu. He described his experiences of déjà vu as extremely distressing. No clear organic cause of the déjà vu was found and Wells et al. (2021) determined that the persistent déjà vu might be psychogenic in nature.

In the current volume, Wells et al. (2021) administered an online questionnaire to a large sample of people with clinical levels of anxiety and controls to test the hypothesis that anxiety provokes déjà vu experiences. They found that their Anxiety Group reported a significantly higher 
frequency of déjà vu episodes over the previous month than controls, experiencing approximately 3 episodes per month, although the groups reported non-significant differences over the past year. People with anxiety reported finding déjà vu episodes significantly more distressing than the Control Group. Perhaps the clearest finding was regarding triggers: people with anxiety were more than twice as likely to report that anxiety triggered a déjà vu experience. Finally, correlational analysis revealed significant correlations between the depression, anxiety and stress and the frequency of déjà vu in the last month and year for both the control group and the people with anxiety.

Finally, we come to the experimental studies which have the aim of understanding the cognitive and neurological processes at play in déjà vu. They take the approach of trying to produce déjà vu in healthy samples in laboratory settings according to current theories of déjà vu formation. Broadly speaking, this approach uses existing memory paradigms to better understand the processes at play in déjà vu, prescience, and jamais vu. Cleary and colleagues (Cleary, McNeelyWhite, Huebert and Claxton, 2021) build on previous work (Cleary \& Claxton, 2018) showing that experimentally-generated déjà vu experiences are associated with stronger feelings of being able to predict what is about to happen (though these feelings of prediction are, of course, unfounded). Across three experiments using immersive navigation videos, they show that choice points for which déjà vu is reported, are associated with greater feelings of being able to predict what will happen next, and with greater levels of familiarity. The link between feelings of familiarity and prediction was also borne out in these data, suggesting an array of epistemic feelings coincide with the déjà vu experience. Cleary and colleagues make clear that such agglomerations of subjective experience are not limited to déjà $\mathrm{vu}$, drawing links with other quirks of memory, such as the tip-of-the-tongue sensation.

A key feature of this work is the emphasis Cleary and colleagues place on the importance of the subjective experience of déjà vu. They recognise that what captures the imagination about the experience is not restricted to what we psychologists have traditionally focused on (i.e. the potential memory error of elevated familiarity), but includes other feelings that flow from, and add to the uncanny nature of déjà vu. This emphasis on the subjective not only adds to our understanding of the qualities of déjà $\mathrm{vu}$, but also opens up new avenues for understanding the diverse range of subjective experiences associated déjà vu (e.g. links between intensity of experience and the feeling of prescience; Brown, 2004) across a range of non-clinical and clinical domains.

In their experimental paper, Urquhart and colleagues (Urquhart, Sivakumaran, Macfarlane and O'Connor, 2021) use a paradigm previously reported to generate reports of déjà vu behaviourally (Urquhart \& O'Connor, 2014), and image participants undergoing the task using functional magnetic resonance imaging (fMRI). They found that activation linked to déjà vu in frontal midline, and parietal brain regions--those typically associated with conflict detection and resolution. The authors infer from these findings that those experiencing déjà vu are not only responding to a feeling of inappropriate familiarity (a conflict between the feeling of familiarity and the awareness that this feeling should not be experienced in the setting in question), but may well be motivated to resolve this when it is set again salient evidence that what is being encountered is in fact novel.

This work highlights the potential utility the development of experimental analogues has, in allowing researchers to go on and explore the déjà vu experience in ways that would be impossible for naturalistic experiences. It also highlights the extent to which neuroimaging, alongside other methodological advancements, has the potential to contextualise subjective experience. In this case evidence for déjà vu being associated with the feeling of conflict between familiarity and salient novelty, as opposed to familiarity alone, points towards explanations for why simple recognition false alarms don't tend to be reported as déjà vu, and potentially moves us towards a tighter definition of the experience.

Finally, Moulin and colleagues (Moulin, Bell, Turunen, Baharin and O'Connor, 2021) investigate jamais vu, proposing that the phenomenon of word alienation resembles the sensation of jamais vu. Although recent research has explored the induction of déjà vu in the laboratory, there has been no such interest in jamais vu, which is surprising since there is a long history of using word alienation tasks to provoke feelings which could be described as similar to the experience of jamais vu. In such tasks, participants report a "loss of meaning" either due to long fixation (e.g. Don \& Weld, 1924) or repetitive writing. According to the earliest descriptions of this experience from the turn of the 19th century, its phenomenology is of strangeness, unfamiliarity and even fear. Moulin et al. (2021) propose that the subjective experience of word alienation is that of "jamais vu", and that the underlying process is that of semantic satiation (see Esposito \& Pelton, 1971, for a review). Unsurprisingly, participants in the two experiments reported in Moulin et al. (2021) reported feeling strange on repeatedly writing the words. Importantly, the frequency of experiences of jamais vu in daily life correlated with the frequency of déjà vu in daily life, and there was some evidence that the susceptibility to jamais vu in the experiment was related to the frequency of such experiences in daily life. The questionnaire part of the study revealed that the jamais vu experience, despite being relatively easy to reproduce in the laboratory, was even less frequent in daily life than déjà vu. 


\section{What comes next in Déjà vu?}

Our goal in this special issue was to present the state of the art of déjà vu research and collate the disparate research approaches together which can be used to study this infrequent and unpredictable phenomenon. In doing so, we hope to generate interest in the topic and outline ways in which the phenomenon can be explored. Clearly a priority is to triangulate on common explanations and mechanisms by using a multimethod approach, and a clear first step might be to combine the experimental and neuropsychological approaches, such that we examine the outcomes of experimentally inducing déjà vu in populations who experience it more or less frequently than controls. Such an approach has already yielded success in examining the tip of the tongue experience, especially concerning the patterns that changes with age or neurodegenerative pathology (e.g. Schwartz \& Frazier, 2005; Souchay \& Smith, 2013). As an example, because the ageing process is related to a reduction in déjà vu experiences according to questionnaire studies (Moulin et al., 2014), it would be of interest to see whether older adults are less susceptible to déjà vu-like feelings in paradigms which are known to generate such feelings. Since a number of different approaches generate déjà vu-like feelings in the laboratory, all of these could be harnessed in the approach used by Urquhart and colleagues in 2021, since we would expect some overlapping functional anatomy in all the different analogues of the déjà vu experience. Likewise, patients with frequent déjà vu experiences or identifiable triggers need to be tested in functional neuroimaging paradigms, where again cerebral regions and networks involved in déjà vu can be more clearly delineated. In terms of questionnaire research, given the availability of theory driven accounts of déjà vu and experimental analogues of déjà vu it appears that a priority would be to develop a tool which reliably and consistently quantifies déjà vu experiences in self report, and perhaps using experience sampling and online means to gather datasets which compensate for the infrequency of the experience with their large scale and ongoing nature.

Beyond being mere curiosities, déjà vu and jamais vu as described here should be harnessed to investigate memory function since they underline the complexity of memory decision making, subjective experience and metacognitive processes. Moreover, in several different populations and aetiologies, déjà vu experiences are debilitating and alienating and thus need to be better understood and treated. In this regard, the symptom education approach to reducing déjà vu symptoms presented in this paper shows the benefits of a theory-based approach and somewhat justifies our metacognitive take on the phenomenon. If we are able to reframe people's metacognitive interpretation of dissociative states such as déjà vu, it is likely to have consequent benefits for wellbeing.
Déjà vu and other dissociative states will continue to be intriguing phenomena that demand our attention. Careful consideration of the processes and plausible mechanisms behind the phenomena is needed, and we have started accumulating datasets to constrain our theorising. Indeed, we shall probably need to gather far more data on déjà vu and similar states before we can incorporate them fully into current neuroscientific accounts of memory. Until now, there has been far too much theorising about déjà vu, without enough actual data. On the one hand, it is excellent that researchers are now bringing déjà vu into mainstream accounts of memory retrieval processes, such as classifying déjà vu as "implicit scene memory" (Rubin, 2021), but without data to support such claims, the mention of déjà vu amounts to little more than academic clickbait.

In 2020, according to the Web of Knowledge, 33 papers were published with déjà vu in the title, but none of these were about déjà vu in memory, psychology or the neurosciences. Instead, the term is used to sum up strange or repetitive fundings. It seems to be a repetitively popular concept, and one which can be used to enliven a dull scientific field or add wit to a critical debate. But even though it is now possible to collect together research articles on déjà vu by internationally recognised scholars, it is still a topic which remains firmly outside the mainstream of cognitive psychology. If, like several other authors before us, we describe it as an illusion, we can only draw embarrassing comparisons with what the study of perception has gained by careful examination of illusions: 38 articles in the Web of Science with "visual illusion" in the title in 2020, including examinations of various clinical populations, neuroimaging, theoretical accounts and even presentations of new kinds of illusions.

Coming full circle, Schacter, in his 2021 update of his notion of the seven sins of memory (Schacter, 2021), acknowledges that memory research has now made inroads into déjà $v u$, even citing research presented in this special issue. This is only made possible by the circular, recursive time-loop that is scientific publishing.

\section{References}

Adachi, N., Adachi, T., Kimura, M., Akanuma, N., Takekawa, Y., \& Kato, M. (2003). Demographic and psychological features of déjà vu experiences in a nonclinical Japanese population. The Journal of nervous and mental disease, 191(4), 242-247. https://doi.org/10. 1097/01.NMD.0000061149.26296.DC

Adachi, T., Adachi, N., Takekawa, Y., Akanuma, N., Ito, M., Matsubara, R., Iked, H., Kimura, M., \& Arai, H. (2006). Déjà vu experiences in patients with schizophrenia. Comprehensive psychiatry, 47(5), 389-393. https://doi.org/10.1016/j.comppsych.2005.12.003

Arnaud, F. L. (1896). Un cas d'illusion du 'déjà vu' ou de 'fausse memoire'. Annales Medico-Psychologiques, 3, 455-471.

Bartolomei, F., Barbeau, E. J., Nguyen, T., McGonigal, A., Régis, J., Chauvel, P., \& Wendling, F. (2012). Rhinal-hippocampal interactions during déjà vu. Clinical Neurophysiology, 123(3), 489-495. https://doi.org/10.1016/j.clinph.2011.08.012 
Bernstein, E. M., \& Putnam, F. W. (1986). Development, reliability, and validity of a dissociation scale. The Journal of Nervous and Mental Disease, 174(12), 727-35. https://doi.org/10.1097/00005053198612000-00004

Bertrand, J. M., Martinon, L. M., Souchay, C., \& Moulin, C. J. (2017). History repeating itself: Arnaud's case of pathological déjà vu. Cortex, 87, 129-141. https://doi.org/10.1016/j.cortex.2016.02.016

Brandt, K. R., Conway, M. A., James, A., \& von Oertzen, T. J. (2021). Déjà vu and the entorhinal cortex: Dissociating recollective from familiarity disruptions in a single case patient. Memory, 29(7), 859-868. https://doi.org/10.1080/09658211.2018.1543436

Brázdil, M., Mareček, R., Urbánek, T., Kašpárek, T., Mikl, M., Rektor, I., \& Zeman, A. (2012). Unveiling the mystery of déjà vu: the structural anatomy of déjà vu. cortex, 48(9), 1240-1243. https://doi.org/10. 1016/j.cortex.2012.03.004

Brown, A. S. (2003). A review of the déjà vu experience. Psychological Bulletin, 129(3), 394-413. https://doi.org/10.1037/0033-2909.129.3.394

Brown, A. S. (2004). The déjà vu experience. Psychology Press.

Brown, A. S., \& Marsh, E. J. (2009). Creating illusions of past encounter through brief exposure. Psychological Science, 20(5), 534-538. https://doi.org/10.1111/j.1467-9280.2009.02337.x

Cleary, A. M. (2008). Recognition memory, familiarity, and déjà vu experiences. Current Directions in Psychological Science, 17(5), 353-357. https://doi.org/10.1111/j.1467-8721.2008.00605.x

Cleary, A. M., \& Claxton, A. B. (2018). Déjà Vu: An Illusion of Prediction. Psychological Science, 29(4), 635-644. https://doi.org/10.1177/ 0956797617743018

Cleary, A. M., McNeely-White, K. L., Huebert, A. M., \& Claxton, A. B. (2021). Déjà vu and the feeling of prediction: An association with familiarity strength. Memory, 29(7), 904-920. https://doi.org/10. 1080/09658211.2018.1503686

Curot, J., Pariente, J., Hupé, J. M., Lotterie, J. A., Mirabel, H., \& Barbeau, E. J. (2021). Déjà vu and prescience in a case of severe episodic amnesia following bilateral hippocampal lesions. Memory, 29(7), 843-858. https://doi.org/10.1080/09658211.2019.1673426

da Sousa, R. (2009). Epistemic feelings. Mind and Matter, 7(2), 139-161.

Don, V. J., \& Weld, H. P. (1924). Lapse of meaning with visual fixation. The American Journal of Psychology, 35(3), 446-450. https://doi.org/ $10.2307 / 1414024$

Ernst, A., Delrue, G., \& Willems, S. (2021). Overcoming familiarity illusions in a single case with persistent déjà vu. Memory, 29(7), 869-883. https://doi.org/10.1080/09658211.2018.1510965

Esposito, N. J., \& Pelton, L. H. (1971). Review of the measurement of semantic satiation. Psychological Bulletin, 75(5), 330-346. https:// doi.org/10.1037/h0031001

Illman, N. A., Butler, C. R., Souchay, C., \& Moulin, C. J. (2012). Déjà experiences in temporal lobe epilepsy. Epilepsy Research and Treatment, 2012, 1-15. https://doi.org/10.1155/2012/539567

Jacoby, L. L., \& Whitehouse, K. (1989). An illusion of memory: False recognition influenced by unconscious perception. Journal of experimental psychology: General, 118(2), 126-135. https://doi.org/10. 1037/0096-3445.118.2.126

Kalra, S., Chancellor, A., \& Zeman, A. (2007). Recurring déjà vu associated with 5-hydroxytryptophan. Acta Neuropsychiatrica, 19(5), 311313. https://doi.org/10.1111/j.1601-5215.2007.00245.x

Martin, C. B., Fiacconi, C. M., \& Köhler, S. (2015). Déjà vu. In Addis D.R Barense M, \& Duarte A (Eds.), The Wiley Handbook on the Cognitive Neuroscience of Memory (pp. 172-189). Hoboken, NJ: Wiley.

Martin, C. B., Mirsattari, S. M., Pruessner, J. C., Burneo, J. G., HaymanAbello, B., \& Köhler, S. (2021). Relationship between déjà vu experiences and recognition-memory impairments in temporal-lobe epilepsy. Memory, 29(7), 884-894. https://doi.org/10.1080/09658211. 2019.1643891

Martin, C. B., Mirsattari, S. M., Pruessner, J. C., Pietrantonio, S., Burneo, J. G., Hayman-Abello, B., \& Köhler, S. (2012). Déjà vu in unilateral temporal-lobe epilepsy is associated with selective familiarity impairments on experimental tasks of recognition memory.
Neuropsychologia, 50(13), 2981-2991. https://doi.org/10.1016/j. neuropsychologia.2012.07.030

Moulin. (2017). The cognitive neuropsychology of déjà vu. Abingdonon-Thames. UK: Routledge.

Moulin, C. J. (2013). Disordered recognition memory: recollective confabulation. Cortex, 49(6), 1541-1552. https://doi.org/10.1016/j. cortex.2013.01.010

Moulin, C. J. A., \& Souchay, C. (2014). Epistemic Feelings and Memory. In Perfect T \& Lindsay S (Eds.), The Sage Handbook of Applied Memory (pp. 520-539). Newbury Park, CA: Sage.

Moulin, C. J., Bell, N., Turunen, M., Baharin, A., \& O'Connor, A. R. (2021). The the the the induction of jamais vu in the laboratory: Word alienation and semantic satiation. Memory, 29(7), 933-942. https://doi.org/10.1080/09658211.2020.1727519

Moulin, C. J., Souchay, C., Bradley, R., Buchanan, S., Karadoller, D. Z., \& Akan, M. (2014). Déjà vu in older adults. In Schwartz B \& Brown A (Eds.), Tip-of-the-tongue states and related phenomena (pp. 281304). Cambridge: Cambridge University Press.

Neppe, V. M. (1983). The psychology of déjà vu: Have I been here before? Witwatersrand University Press.

Nyberg, L. (2005). Any novelty in hippocampal formation and memory? Current opinion in neurology, 18(4), 424-428. https:// doi.org/10.1097/01.wco.0000168080.99730.1c

Penfield, W., \& Perot, P. (1963). The brain's record of auditory and visual experience: a final summary and discussion. Brain, 86(4), 595-696. https://doi.org/10.1093/brain/86.4.595

Pešlová, E., Mareček, R., Shaw, D. J., Kašpárek, T., Pail, M., \& Brázdil, M. (2018). Hippocampal involvement in nonpathological déjà vu: Subfield vulnerability rather than temporal lobe epilepsy equivalent. Brain and behavior, 8(7), e00996. https://doi.org/org/10.1002/ brb3.996

Rubin, D.C. (2021). A conceptual space for episodic and semantic memory. Memory \& Cognition. https://doi.org/10.3758/s13421021-01148-3

Schacter, D. L. (2002). The seven sins of memory: How the mind forgets and remembers. Houghton Mifflin Company.

Schacter, D. L. (2021). The seven sins of memory: an update. Memory, 1-6. https://doi.org/10.1080/09658211.2021.1873391

Schwartz, B. L., \& Frazier, L. D. (2005). Tip-of-the-tongue states and aging: Contrasting psycholinguistic and metacognitive perspectives. The Journal of General Psychology, 132(4), 377-391. https:// doi.org/10.3200/GENP.132.4.377-391

Schwartz, B. L., \& Metcalfe, J. (2011). Tip-of-the-tongue (TOT) states: Retrieval, behavior, and experience. Memory \& cognition, 39(5), 737-749. https://doi.org/10.3758/s13421-010-0066-8

Singh, S. (2007). Adolescent salvia substance abuse. Addiction, 102(5), 823-824. https://doi.org/10.1111/j.1360-0443.2007.01810.x

Sno, H. N., Schalken, H. F., de Jonghe, F., \& Koeter, M. W. (1994). The Inventory for déjà vu Experiences Assessment. Development, Utility, Reliability, and Validity. The Journal of nervous and mental disease, 182(1), 27-33. https://doi.org/10.1097/00005053-19940 1000-00006

Souchay, C., \& Smith, S. J. (2013). Subjective states associated with retrieval failures in Parkinson's disease. Consciousness and cognition, 22(3), 795-805. https://doi.org/10.1016/j.concog.2013. 05.001

Urquhart, J. A., \& O'Connor, A. R. (2014). The awareness of novelty for strangely familiar words: a laboratory analogue of the déjà vu experience. PeerJ, 2, e666. https://doi.org/10. 7717/peerj.666

Urquhart, J. A., Sivakumaran, M. H., Macfarlane, J. A., \& O'Connor, A. R. (2021). fMRl evidence supporting the role of memory conflict in the déjà vu experience. Memory, 29(7), 921-932. https://doi.org/10. 1080/09658211.2018.1524496

Warren-Gash, C., \& Zeman, A. (2014). Is there anything distinctive about epileptic déjà vu? Journal of Neurology, Neurosurgery \& Psychiatry, 85 (2), 143-147. https://doi.org/10.1136/jnnp-2012-303520 
Wells, C. E., O'Connor, A. R., \& Moulin, C. J. (2021). Déjà vu experiences in anxiety. Memory, 29(7), 895-903. https://doi.org/10.1080/ 09658211.2018 .1538418

Wells, C. E., Moulin, C. J., Ethridge, P., Illman, N. A., Davies, E., \& Zeman, A. (2014). Persistent psychogenic déjà vu: a case report. Journal of Medical Case Reports, 8(1), 1-4. https://doi.org/10.1186/1752-1947-8-414

Akira R. O'Connor University of Saint Andrews, Scotland (iD) http://orcid.org/0000-0002-7943-5183
Christine Wells

Leeds Trinity University, England (D) http://orcid.org/0000-0002-7148-0318

Chris J. A. Moulin Université Grenoble Alpes, France and

Institut Universitaire de France

christopher.moulin@univ-grenoble-alpes.fr 